\title{
On Typhoon Track Deflections near the East Coast of Taiwan
}

\author{
Li-HuAN HSU \\ Taiwan Typhoon and Flood Research Institute, National Applied Research Laboratories, Taipei, Taiwan
}

SHIH-HAO SU

Department of Atmospheric Sciences, Chinese Culture University, Taipei, Taiwan

ROBERT G. FOVELL

Department of Atmospheric and Environmental Sciences, University at Albany, State University of New York, Albany, New York

HuNG-CHI KUO

Department of Atmospheric Sciences, National Taiwan University, Taipei, Taiwan

(Manuscript received 18 July 2017, in final form 12 March 2018)

\begin{abstract}
Typhoons with "deflection tracks" (DTs) within a 200-km distance of the mountainous island of Taiwan are examined. We analyze 84 landfalling typhoons that compose 49 DT cases turning to the left-hand side, including 18 with very large deflection angles $\left(\mathrm{DA}>20^{\circ}\right)$ and another 7 having looped tracks (LTs). Most of the large DA and LT cases are "northern landfall" type, reaching Taiwan's east coast poleward of $24^{\circ} \mathrm{N}$ and originally possessing relatively slow translation speeds $\left(\sim 4 \mathrm{~m} \mathrm{~s}^{-1}\right)$. Their average translation speeds, however, increase by $50 \%$ in the $3 \mathrm{~h}$ prior to landfall. The WRF Model is used to simulate DT cases, and potential vorticity (PV) tendency diagnosis is used to interpret the contributions of the horizontal advection (HA), vertical advection (VA), and diabatic heating (DH) terms. The northern landfall tropical cyclones (TCs) possess significant cross-mountain flow to the south of the storm near the coast, resulting in vorticity stretching (the VA effect) and subsidence warming. The subsidence suppresses storm convection and produces heating asymmetries (the DH effect) that can induce significant southwestward deflections. The cross-mountain VA and DH effects are weaker for the "southern landfall" storms. The results explain well the observed increase of translation speed prior to landfall in DT cases and show that the HA effect, in general, does not contribute to the track deflection. Our results highlight the impact of topography on TC track by the vorticity stretching effect and by asymmetric diabatic heating.
\end{abstract}

\section{Introduction}

There are many previous observational and numerical studies indicating that the tracks of tropical cyclones (TCs) may be altered while approaching the Central Mountain Range (CMR) of Taiwan (e.g., Brand and Blelloch 1974; Wang 1980; Bender et al. 1987; Yeh and Elsberry 1993). Westbound TCs sometimes start exhibiting a southward turn at a distance of around $200 \mathrm{~km}$ offshore and then make landfall or deflect southward around Taiwan (Brand and Blelloch 1974; Wang 1980; Yeh and Elsberry 1993). We term this phenomenon a "deflection track" (DT). Previous numerical simulations show that some DTs are

\footnotetext{
Corresponding author: Shih-Hao Su, ssh3@g.pccu.edu.tw
}

caused by the orographic blocking effect, which means the environmental flow is distorted or impeded by the Taiwan topography, thereby modifying the TC structure. Specifically, an asymmetric flow across the typhoon center is generated when a TC moves near the topography that sometimes leads to a southward deflection for storms approaching the northern part of the island (Bender et al. 1987; Yeh and Elsberry 1993; Lin et al. 1999).

Lin et al. (2005) conducted a series of numerical experiments of a vortex across an idealized mesoscale mountain to identify the relevant nondimensional dynamical parameters. They suggest that DTs within a 200-km distance offshore preferentially occur with smaller values of the vortex Froude number $\left(\mathrm{VFr}=V_{\max } / N h\right)$ and the vortex/mountain size parameter $\left(R_{\max } / L_{y}\right)$, 
where $V_{\max }$ is the vortex maximum wind, $N$ is buoyancy frequency, $h$ is mountain height, $R_{\max }$ is vortex radius of $V_{\max }$, and $L_{y}$ is the mountain's latitudinal extent. The degree of track deflection is controlled predominantly by the steering flow Froude number $(U / N h)$, where $U$ is the mean flow speed. In the situation of strong blocking (i.e., the steering flow Froude number is small), a northerly asymmetric flow between the parent cyclone and the mountain range develops that modifies the cyclone motion. Owing to vorticity advection, the vortex is deflected toward the south by the mean flow near the mountain coast. This kind of channeling effect is also found in idealized experiments and simulations of Typhoons Haitang (2005) and Krosa (2007) (Jian and Wu 2008; Huang et al. 2011; Wu et al. 2015). However, there are some TC cases with northward deflection tracks, and these have been discussed in previous observations and numerical studies (Brand and Blelloch 1974; Wang 1980; Chang 1982; Bender et al. 1987; Yeh and Elsberry 1993; Lin et al. 2005). They suggest that the northward deflection track was also due to either terrain-altered environmental flow or the terrain blocking effect in certain scenarios.

The vorticity stretching effect associated with the flow across the steep terrain may also lead to DTs for westward-moving typhoons (Kuo et al. 2001). Vorticity stretching is important when the typhoon is strong and VFr is large so that the flow can cross the topography with less blocking. In such a case, the cyclone will have a local northwest motion following the direction of local topographic potential vorticity (PV) gradient, which is similar to the $\beta$ effect (Carnevale et al. 1988, 1991). The topographic $\beta$ effect affecting TC motion in the Sierra Madre has been reported in Zehnder (1993).

Kuo et al. (2001) demonstrated that as the typhoon circulation crosses the CMR of Taiwan, vorticity stretching leads to the southward shift of typhoon motion before landfall. Idealized experiments have shown that the location where vorticity stretching occurs will depend on the relative size and location of the typhoons and topography (Lin et al. 2005; Huang and Lin 2008; Lin and Savage 2011; Liu et al. 2016; Lin et al. 2016). The vorticity budget analysis showed that the track deflection upstream is dominated by vorticity stretching occurring on the east side of the CMR in the cases in which the vortex is small, compared to the mountain size, or is located at the northern portion of the CMR (Lin and Savage 2011). The vorticity stretching on the lee side may be associated with significant subsidence. Chen et al. (2010) studied the subsidence warming due to the TC flow crossing the mountain in Taiwan, and their results indicate that when typhoons make landfall in northern Taiwan, significant surface warming due to the subsidence usually appears at the southeastern coast, owing to cross-mountain downslope winds. The observed subsidence warming and its associated vorticity stretching on the lee side of the CMR may lead to the DTs of typhoons.

In addition to its mechanical effects, topographically modulated diabatic heating may also play an important role in influencing TC motion (Chang 1982; Chan et al. 2002; Wang et al. 2012, 2013). Topographically phaselocked, convective diabatic heating induced by the TC circulation impacting the CMR can significantly alter the TC track (Hsu et al. 2013, hereafter H13; Tang and Chan 2014, 2015). H13 also suggested the diabatic heating may contribute to causing track deflections before landfall. All the physical processes addressed so far, including the terrain blocking, the vorticity stretching, and the diabatic heating, can be consistently diagnosed via PV tendency analysis (cf. Wu and Wang 2000, 2001), which has proven to be a useful way of understanding typhoon motion (e.g., Fovell et al. 2010; Cao et al. 2011; Tang and Chan 2014, 2015). The cyclone tends to move toward the direction where the positive wavenumber-1 component (WN1) of the PV tendency is dominant.

Among the previous numerical studies regarding terraininduced DTs near Taiwan, the majority have focused on either westward-moving TC cases with looped tracks (LTs) or discussed TC deflection mechanisms within idealized frameworks (Lin et al. 2005; Lin and Savage 2011; Liu et al. 2016; Lin et al. 2016; Tang and Chan 2016). Each appears to emphasize a different mechanism related to the terrain effect that can produce DTs near the Taiwan coast. Moreover, there appears to be a lack of climatological analysis of DTs near Taiwan's east coast. Therefore, in this study, we perform an observational analysis of 84 typhoons that reached the east coast of Taiwan during the 51-yr period between 1960 and 2010 (inclusive). Idealized numerical simulations with realistic Taiwan terrain profile employing PV tendency diagnoses are then used to interpret the observational results.

The organization of this paper is as follows. Section 2 presents observational statistics of typhoon track deflections before landfall. Section 3 describes the Weather Research and Forecasting (WRF) Model experiments and the PV tendency diagnosis. The WRF simulations and the PV tendency analyses are presented in section 4. Section 5 gives the conclusions.

\section{Observation}

This study focuses on typhoons approaching within a $200-\mathrm{km}$ distance of Taiwan's east coast. Of the 96 typhoons that occurred in the 51-yr period spanning 1960-2010, 84 eventually made landfall on the east coast of Taiwan after an average interval of about $12 \mathrm{~h}$. Some 
(a)

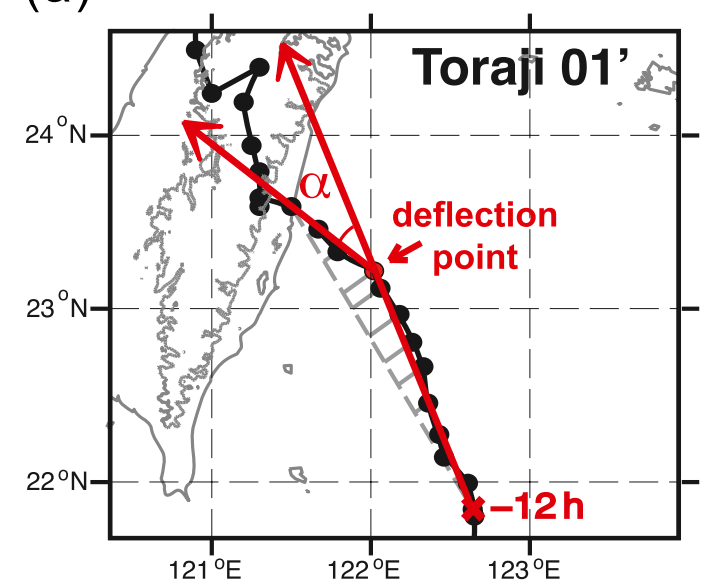

(b)

(c)
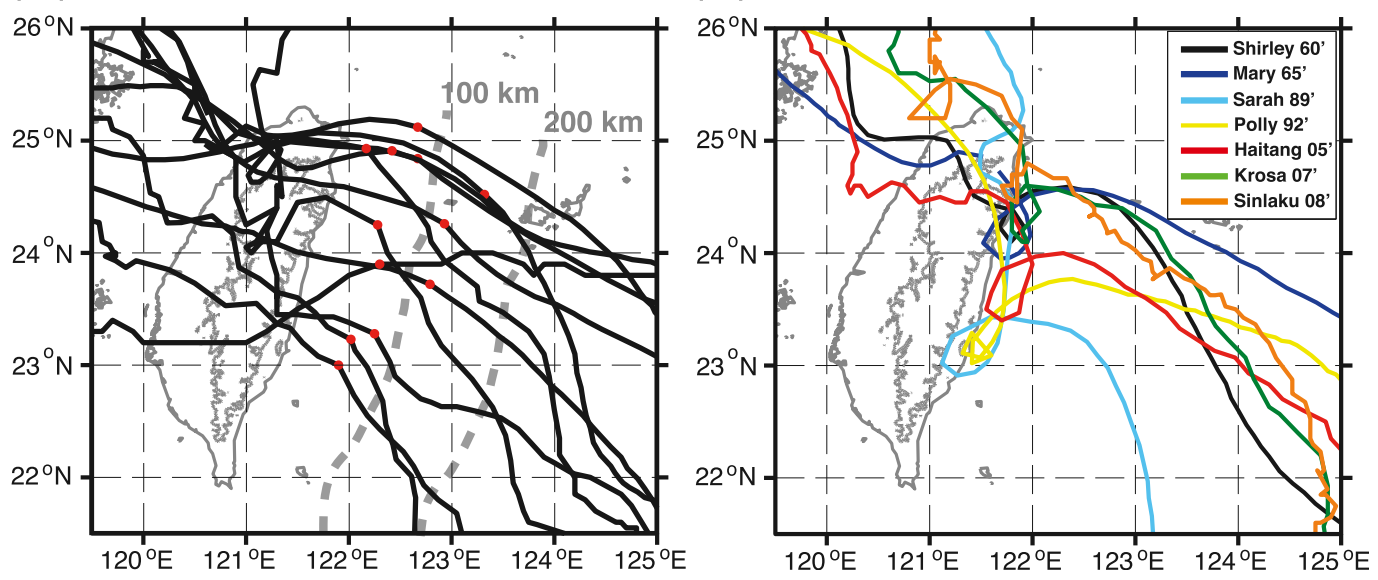

FIG. 1. (a) Determination of the deflection angle $\alpha$ for Typhoon Toraji (2001). The deflection point was defined as the location at which the TC had the longest interval of linear motion within the 12-h period before landfall. The included angle of those two vectors is defined as $\alpha$. (b) Examples of DT typhoons with DA greater than $20^{\circ}$. The positions of deflection points are marked as red dots. (c) Tracks for seven LT typhoons.

of these typhoons also exhibited track variations or deflections as they approached the island that can be described as turning to the left-hand side and right-hand side (rhs), relative to their motions.

Figure 1 presents example DT storms that possess large deflection angles (DAs) $\left(\alpha>20^{\circ}\right)$ or LTs. Our method of computing the storm track DA $\alpha$, illustrated in Fig. 1a, utilized hourly position information from the Central Weather Bureau (CWB) typhoon database (Wang 1980; Shieh et al. 1998) that was augmented by radar and satellite data from 1977 onward. To measure TC deflection quantitatively, we identified the deflection point as the location at which the TC had the longest interval of straight motion within the 12 -h period before landfall. Then, the DA $\alpha$ is formed between the original and deflected TC motion vectors. The typhoon exhibits left-hand (right hand) turning when the DA is positive (negative), and its magnitude indicates the amplitude of the deflection. If the typhoon position is farther than $200 \mathrm{~km}$ from the east coast of Taiwan at $12 \mathrm{~h}$ before landfall $(t=-12 \mathrm{~h})$, the position where the storm first reaches $200 \mathrm{~km}$ is selected instead.

Table 1 categorizes the 84 typhoon cases into subgroups based on DA magnitude and the latitude of the deflection point, and it reveals that the track deflection is influenced by the position of the typhoon relative to the Taiwan topography. Typhoons tend to turn to the righthand $\left(\alpha \leq 0^{\circ}\right)$ and left-hand $\left(\alpha>0^{\circ}\right)$ sides while approaching southern and northern Taiwan, respectively. For the 35 cases with negative $\alpha, 23$ (66\%) had deflection points south of $23^{\circ} \mathrm{N}$ latitude. Of the remaining 49 storms, 18 possessed $\alpha>20^{\circ}$, with the majority ( $90 \%$; 16 of 18) located north of $23^{\circ} \mathrm{N}$. There were also 7 typhoons exhibiting LTs prior to landfall, all occurring 
TABLE 1. Numbers of typhoons with different DAs categorized by latitude range of their deflection point. DA greater than zero represents the typhoon cases with DTs turning to the left-hand side, and LTs are the looped track typhoon cases.

\begin{tabular}{lccccc}
\hline \hline Category (No.) & $\alpha \leq 0^{\circ}(35)$ & $0^{\circ}<\alpha \leq 10^{\circ}(9)$ & $10^{\circ}<\alpha \leq 20^{\circ}(15)$ & $20^{\circ}<\alpha(18)$ & LT (7) \\
\hline North of $24^{\circ} \mathrm{N}(23)$ & 3 & 1 & 4 & 10 & 5 \\
$23^{\circ}-24^{\circ} \mathrm{N}(25)$ & 9 & 3 & 5 & 6 & 2 \\
$22^{\circ}-23^{\circ} \mathrm{N}(23)$ & 13 & 4 & 4 & 2 & 0 \\
South of $22^{\circ} \mathrm{N}(13)$ & 10 & 1 & 2 & 0 & 0 \\
\hline
\end{tabular}

poleward of $23^{\circ} \mathrm{N}$ (Fig. 1c). LT behavior can prolong the duration time prior to landfall and lead to extreme rainfall events in Taiwan (Su et al. 2012; Chang et al. 2013).

If only the mean flow blocking by topography is considered, the westbound typhoons should turn to the south for typhoons approaching southern Taiwan. Previous numerical studies using idealized topography (a Gaussian hill) have shown that the most significant terrain blocking and channeling effects occur near the central peak (Lin et al. 1999; Liu et al. 2016). The storm intensity and translation speed also affect the TC interaction with the topography, but TCs approaching different parts of the topography would have different responses (Yeh and Elsberry 1993; Huang et al. 2011). Although the topography is asymmetric, Taiwan's highest mountain, Yushan, is located around $23.5^{\circ} \mathrm{N}$, and, as a consequence, cases with large DAs should be more likely to have landfalls south of $23.5^{\circ} \mathrm{N}$. However, our analysis indicates that most of the large DA cases occur north of $24^{\circ} \mathrm{N}$ and decrease equatorward, which implies that the terrain blocking is either not always present or at least is not the sole mechanism influencing DT behavior. We hypothesize that the vorticity stretching effect may also be a factor, and strong subsidence south of the typhoon may occur while the storm is approaching northern Taiwan. The downslope wind at the lee side may not only contribute to the vorticity budget, but also suppress convective activity equatorward of the typhoon center.

Figure 2 shows four westward-moving typhoon cases belonging to the positive DA or LT classes. The radar analysis from the Quantitative Precipitation Estimation and Segregation Using Multiple Sensor (QPESUMS) system (Chiou et al. 2006) reveals the appearance of relatively weak convection zones on the southwest sides of these typhoons as they approached the east coast of Taiwan. Chen et al. (2010) suggested that the weak radar reflectivity zones in Typhoons Haitang (2005), Talim (2005), and Krosa (2007) were related to the subsidence warming that occurred when the typhoon's cyclonic circulation was directed eastward, across the CMR. Figure $2 \mathrm{~d}$ also shows similar weak reflectivity zones appeared in Typhoon Saola (2012), during which the CWB also recorded Foehn-type wind events in southeast Taiwan. Therefore, the weak convection zones appearing in the composite radar reflectivity may be evidence for the cross-mountain flow-induced vorticity stretching on the lee side. The vorticity stretching effect can cause DTs when the cross-mountain flow appears near the typhoon (Kuo et al. 2001).

Figure 3 displays the mean translation speed every $3 \mathrm{~h}$ prior to typhoon landfall for LTs and positive DA cases with deflection points located north of $23^{\circ} \mathrm{N}$. Both the LT and large DA $\left(\alpha>20^{\circ}\right)$ cases have relatively slow mean translation speeds at $12 \mathrm{~h}$ before landfall but then experience a significant acceleration as the storms approach the terrain. For the cases with $\alpha$ exceeding $20^{\circ}$, the average $t=-9$ to $-12 \mathrm{~h}$ translation speed is about $5 \mathrm{~m} \mathrm{~s}^{-1}$, while for LT cases, it is about $4 \mathrm{~m} \mathrm{~s}^{-1}$. The average translation speeds $9-12 \mathrm{~h}$ before landfall for LT cases are slower than for the small DA $\left(\alpha<20^{\circ}\right)$ cases. The average speed increases by about $1(2.4) \mathrm{m} \mathrm{s}^{-1}$ for large DA (LT) cases in the $3 \mathrm{~h}$ prior to landfall. These speed increases may cause southward displacements of about $11-26 \mathrm{~km}$ prior to landfall.

In this study, the effects of both diabatic heating and mechanical forcing on DTs will be studied with numerical experiments. The reason why most large DA cases have occurred poleward of $24^{\circ} \mathrm{N}$ will be addressed in following sections.

\section{Model settings and potential vorticity tendency diagnosis}

\section{a. Model settings}

A modified variant of the WRF Model (version 3.1.1; see Fovell and Su 2007; Fovell et al. 2009, 2010; Cao et al. 2011; Hsu et al. 2013) is used in this study. The basic state consists of Jordan's (1958) Caribbean hurricane season composite temperature and humidity sounding with a uniform sea surface temperature (SST) of $29^{\circ} \mathrm{C}$. The Yonsei University (YSU) planetary boundary layer scheme (Hong 2010), Lin et al. (1983) microphysics scheme, RRTM (Mlawer et al. 1997) and Dudhia (1989) radiation packages, and Smagorinsky (horizontal) subgrid-scale mixing scheme are chosen for these idealized simulations. The model domain is square, $1500 \mathrm{~km}$ on a side, with horizontal resolution of $5 \mathrm{~km}$. The model employs 35 vertical layers, topping out at $10 \mathrm{hPa}$. 
(a) 2140 UTC 17 JUL 2005

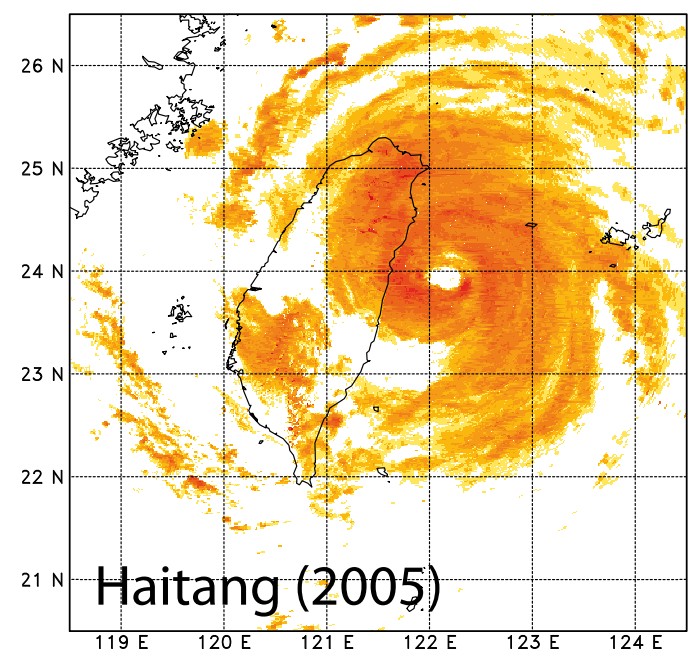

(c) 0650 UTC 06 OCT 2007

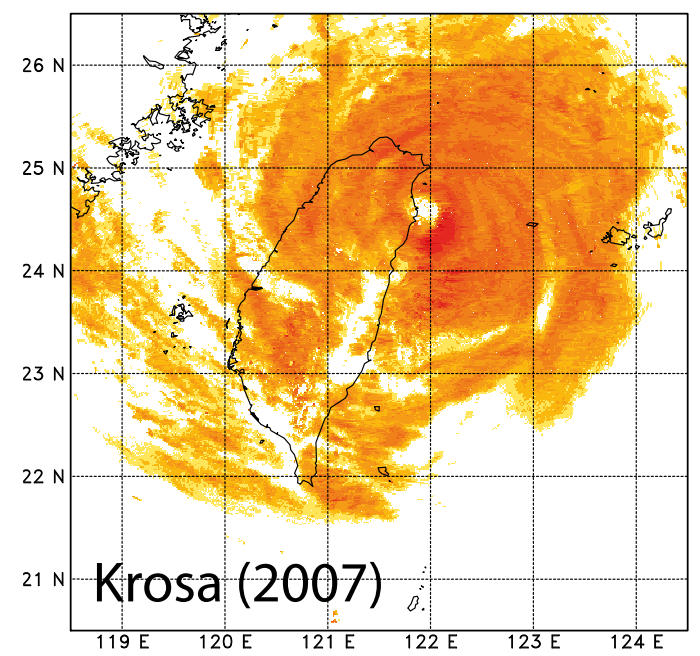

(b) 1430 UTC 31 AUG 2005

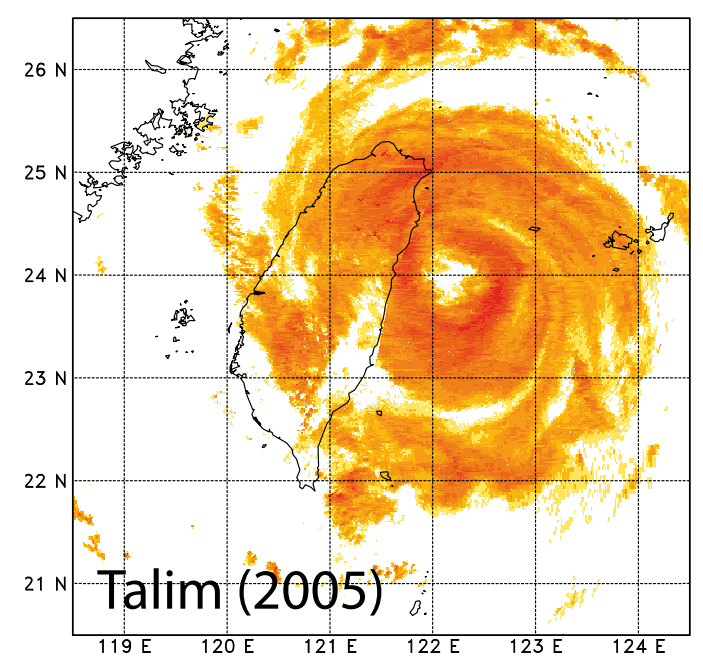

(d) 1730 UTC 01 AUG 2012

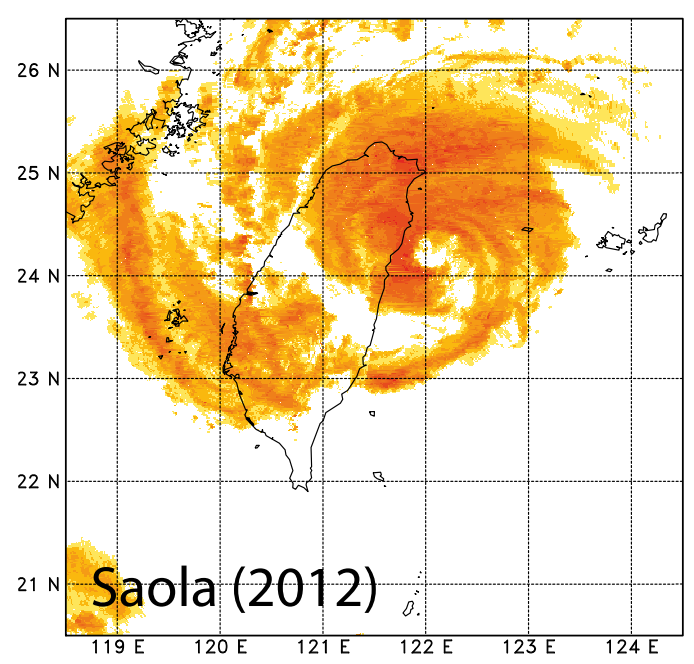

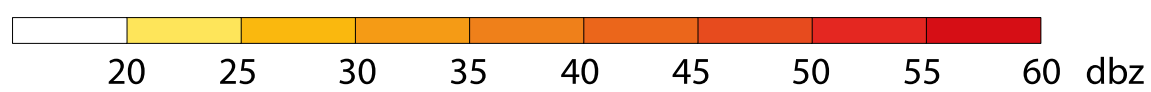

FIG. 2. Composite radar reflectivity from Taiwan CWB's QPESUMS system for (a) Typhoon Haitang (2005),

(b) Typhoon Talim (2005), (c) Typhoon Krosa (2007), and (d) Typhoon Saola (2012).

Our experiments are initialized with an easterly flow that is uniform in both the horizontal and vertical directions and with a magnitude that is varied in the sensitivity experiments. Following H13, the model domain has no topography other than Taiwan, which was constructed from the 30 -arc-s (about $1 \mathrm{~km}$ ) resolution USGS database. As in H13, we supply the island grid points with the same surface roughness and moisture availability as the ocean to focus on the effect of topographically induced asymmetric flow and convection on typhoon motion. Although there is no land, we will employ the term "landfall" to describe the TC encounter with the Taiwan topography. Tropical cyclones were inserted into the initial state via the WRF bogus scheme and initially positioned $400 \mathrm{~km}$ from the east coast of Taiwan.

\section{b. Potential vorticity tendency diagnosis}

The PV tendency diagnosis ( $\mathrm{Wu}$ and Wang 2000, 2001) is used in an attempt to separate the influences of advection and diabatic heating on tropical cyclone motion. As in Fovell et al. (2010, 2016), Cao et al. (2011), 


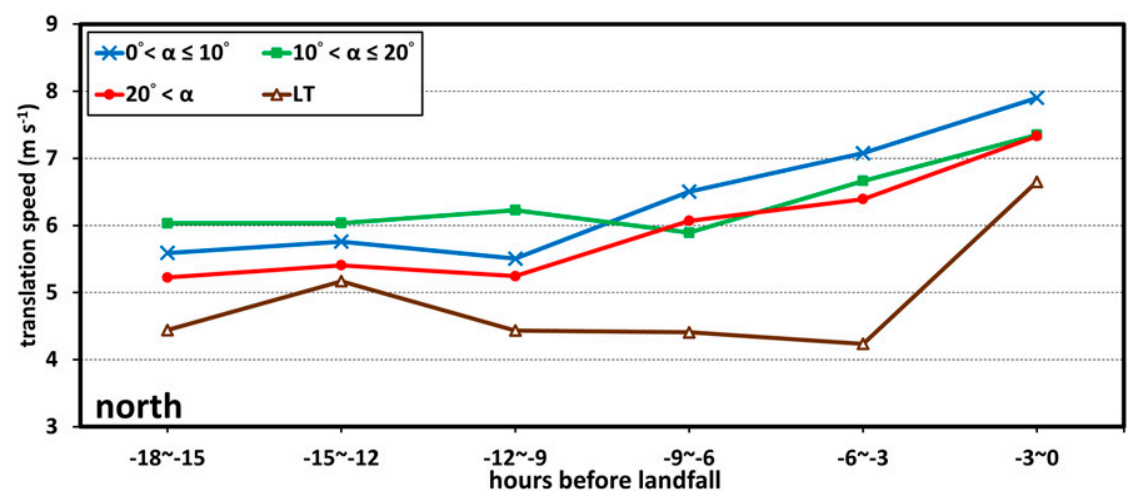

FIG. 3. The 3-hourly mean translation speeds prior to landfall for LT and DT typhoons with positive deflection angles $\alpha$ and deflection points located north of $23^{\circ} \mathrm{N}$. Brown line denotes the translation speed of LT cases. Red, green, and blue lines represent the translation speeds for the $20^{\circ}<\alpha, 10^{\circ}<\alpha \leq 20^{\circ}$, and $0^{\circ}<\alpha \leq 10^{\circ}$ deflection angle subgroups, respectively. The standard deviations in each of the curves are about $2 \mathrm{~m} \mathrm{~s}^{-1}$.

and H13, we employ this analysis in a fundamentally qualitative and comparative fashion in order to help elucidate important differences among time periods and simulations. First of all, the storm motion is correlated with the WN1 component of PV tendency:

$$
\left(\frac{\partial P}{\partial t}\right)_{1}=-C_{x} \frac{\partial P_{s}}{\partial x}-C_{y} \frac{\partial P_{s}}{\partial y},
$$

with

$$
P=-g\left[(\zeta+f) \frac{\partial \theta}{\partial p}-\frac{\partial v}{\partial p} \frac{\partial \theta}{\partial x}+\frac{\partial u}{\partial p} \frac{\partial \theta}{\partial y}\right]
$$

where $P$ is the $\mathrm{PV}, \theta$ is potential temperature, $\zeta$ is the relative vorticity, $g$ is the gravity acceleration, and $f$ is the planetary vorticity. In (1), the subscripts " 1 " and " $s$ " denote the WN1 component and the symmetric part of the vortex $\mathrm{PV}$, and $C_{x}$ and $C_{y}$ are the scalar speeds in the east and north directions, respectively.

The inviscid PV tendency equation can be expanded as

$$
\frac{\partial P}{\partial t}=-\left(u \frac{\partial P}{\partial x}+v \frac{\partial P}{\partial y}\right)-w \frac{\partial P}{\partial z}+\mathrm{DH},
$$

with

$\mathrm{DH}=\frac{1}{\rho}\left[(\zeta+f) \frac{\partial Q}{\partial z}+\left(\frac{\partial u}{\partial z}-\frac{\partial w}{\partial x}\right) \frac{\partial Q}{\partial y}+\left(\frac{\partial w}{\partial y}-\frac{\partial v}{\partial z}\right) \frac{\partial Q}{\partial x}\right]$,

where $u, v$, and $w$ are horizontal and vertical velocities, $\rho$ is density, and $Q$ is the diabatic heating rate. The net $\mathrm{PV}$ tendency is determined by the three terms on the rhs of (3): horizontal advection (HA), vertical advection
(VA), and the diabatic heating term (DH) that depends on gradients of $Q$. H13 determined that the DH term was dominated by vertical gradients of $Q$, which, owing to absolute vorticity being positive, creates $\mathrm{PV}$ when heating increases with height. Note that while $Q$ generates $\mathrm{PV}$, and the translation speed and direction may be altered by $Q$, storms do not move directly toward the region of maximum $Q$. Instead, they translate in the direction where the WN1 PV tendency is positive, and asymmetric diabatic heating can contribute significantly to that. However, the DH term may be a minor factor in storm motion not because there is little diabatic heating in the storm, or because there are no gradients in the heating field, but rather because the heating field itself is relatively symmetric (cf. Fovell et al. 2010; H13).

H13 (p. 1013) discussed some limitations of the PV diagnosis method, including the lack of independence among the HA, VA, and DH terms and their tendency to shift magnitude and orientation with height. These were addressed by averaging the terms over a deep layer (1-11 km above the surface). In our experience, averaging over an appreciable depth above the boundary layer helps to significantly decrease the magnitude of the residual term, which means the combined rhs PV tendency is consistent with the TC moving direction and speed. While we employ PV analysis to facilitate qualitative comparisons, we recognize that the residual term should be small to support the efficacy of the method (and is small in all applications discussed herein). Furthermore, as we focus on persistent, rather than instantaneous, deflections in TC motion, we also employ temporal $(3 \mathrm{~h})$ averaging to smooth out the short-term or random TC motions. We compute the PV tendency terms within a $40000 \mathrm{~km}^{2}$ square horizontal area centered on the TC. In our experiments, this area contains 
the asymmetric PV tendencies that can potentially interact with the symmetric part of TC vortex based on (1).

\section{Numerical results}

\section{a. Overview}

Figure 4 presents the typhoon tracks from experiment U3N (blue), which makes landfall north of $23^{\circ} \mathrm{N}$ (see Table 2), and the corresponding oceanic control (OC; gray) run that has no topography. The initial vortices were placed at $20.25^{\circ} \mathrm{N}, 124.35^{\circ} \mathrm{E}, 400 \mathrm{~km}$ southeast of Taiwan, within a uniform $3 \mathrm{~m} \mathrm{~s}^{-1}$ easterly (U3) flow. Hourly tracks were determined by the local minimum of surface circulation near the location of lowest sea level pressure. Both typhoons initially tracked northwestward at about $3.6 \mathrm{~m} \mathrm{~s}^{-1}$, a consequence of the mean steering and the beta effect, albeit about one-half standard deviation slower than all the categories show in Fig. 3.

After simulation hour 21 (about $17 \mathrm{~h}$ prior to its landfall), however, the translation speed of case U3N started fluctuating with time. In contrast to the OC typhoon, which continued moving northwestward, the U3N track deflected leftward (cyclonically, to the southwest) $3 \mathrm{~h}$ prior to making landfall at simulation hour 38. The 3-h mean translation speeds also increased by $20 \%$, from 3.6 to $4.3 \mathrm{~m} \mathrm{~s}^{-1}$, during the $15-\mathrm{h}$ period preceding the storm reaching the coast. Case U3N's track deflection became as large as $26 \mathrm{~km}$ relative to $\mathrm{OC}$ prior to landfall, which is reasonable, according to Fig. 3 .

Figure 4 also illustrates total rainfall for case U3N between prelandfall simulation hours 35-38 (top) and postlandfall simulation hours 51-54 (bottom); those times are identified by blue crosses superposed on the U3N track. Prior to landfall, the rainfall maximum was located on the windward side of eastern Taiwan. As the typhoon moved to Taiwan's western coast after landfall (Fig. 4b), two rainfall peaks appeared on the CMR's western slope at about $24.1^{\circ}$ and $23.2^{\circ} \mathrm{N}$. The vertical cross sections of temporally averaged diabatic heating $Q$ in Fig. 5 are taken across local rainfall maxima, as indicated in Fig. 4. The distinct wind shift in Figs. 5a and 5b indicates the different windward side of the mountain when the typhoon is prior to landfall and departure, respectively. While most of the heating was found below $5-\mathrm{km}$ altitude prior to landfall (Fig. 5a), the convection was deeper (extending up to $10 \mathrm{~km}$ ) and more vigorous when the typhoon was located in western Taiwan (Fig. 5b).

\section{b. PV tendency analysis for DTs before landfall}

Figure 6 presents PV tendency diagnoses representing two different times for U3N and its southern landfall counterpart, U3S, which was created by positioning an otherwise identical vortex farther to the south (Table 2).

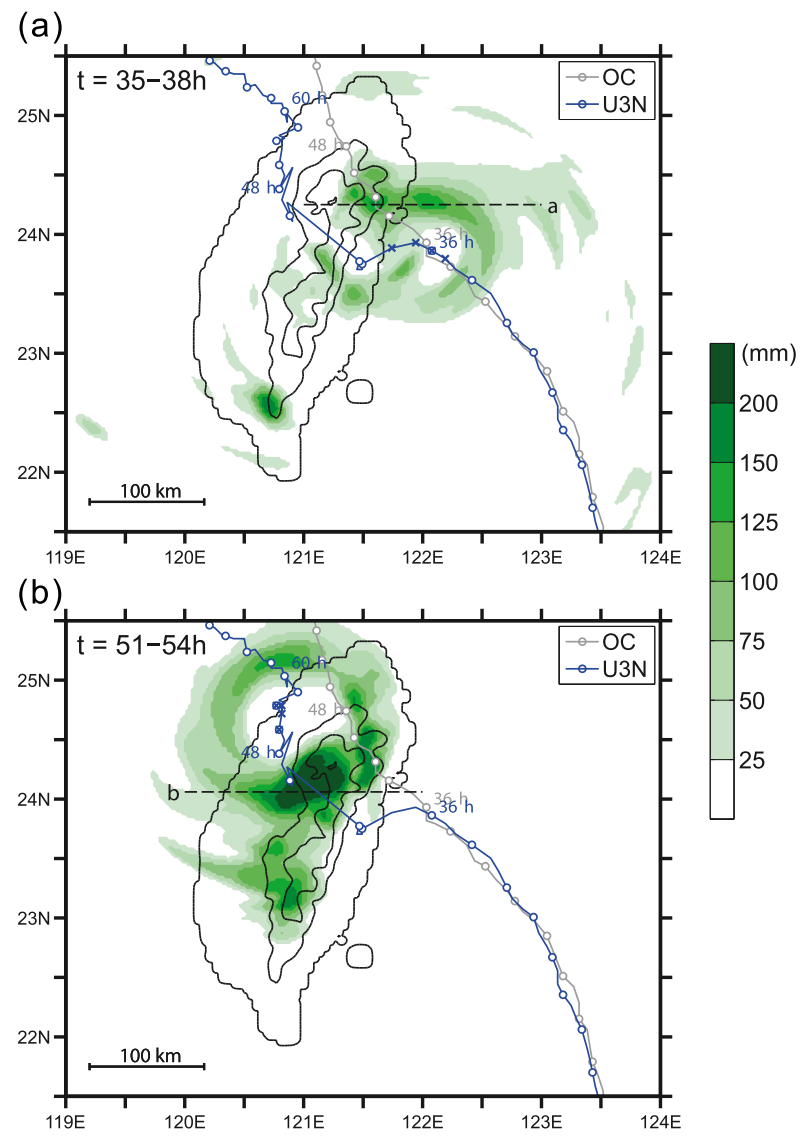

FIG. 4. Typhoon tracks over $72 \mathrm{~h}$ for the Taiwan terrain (U3N; blue) and OC (gray) experiments having initial $3 \mathrm{~m} \mathrm{~s}^{-1}$ easterly mean flow. Three-hourly track positions (circles) are defined by the local surface wind minimum near the sea level pressure minimum. Rainfall totals for U3N over (a) 35-38 and (b) 51-54 h are shaded; these times are indicated by the blue crosses superposed on the U3N track. Dashed lines indicate locations of the cross sections presented in Fig. 5. The topography contour interval is $1000 \mathrm{~m}$. Only part of the $1500 \mathrm{~km}^{2}$ domain is shown.

Note that while U3N presented a significant leftwardturning DT prior to landfall, U3S proceeded northwestward without deviation right up until reaching the coast. The 3-h composite PV tendency contributions of $\mathrm{HA}, \mathrm{VA}$, and DH are denoted as vectors in blue, green, and red, respectively, and the OC case's HA vector is dashed blue. Times referenced are relative to (and negative, prior to) the respective landfalls of the U3N and U3S cases, and the diagnoses represent hours -11 to -8 and -3 to 0

For the OC case, the HA vector magnitude was about $3.2 \mathrm{~m} \mathrm{~s}^{-1}$ and directed northwest, accounting for nearly all of this case's translation. The other terms (not shown) were small, not because vertical advection and diabatic heating were not present, but rather because their WN1 projections were not significant. In contrast, the U3N 
TABLE 2. List of experiments (see text).

\begin{tabular}{lccclcc}
\hline \hline Name & $V_{\max }\left(\mathrm{m} \mathrm{s}^{-1}\right)$ & $R_{\max }(\mathrm{km})$ & $U\left(\mathrm{~m} \mathrm{~s}^{-1}\right)$ & Topography & Diabatic heating & Position \\
\hline U3N & 50 & 50 & -3 & Taiwan & On & North \\
U3S & 50 & 50 & -3 & Taiwan & On & South \\
OC & 50 & 50 & -3 & None & On & North \\
NDH & 50 & 50 & -3 & Taiwan & Off & - \\
OCNDH & 50 & 50 & -3 & None & Off & On \\
U3NV15 & 15 & 50 & -3 & Taiwan & On & North \\
U3NR100 & 50 & 100 & -3 & Taiwan & On & North \\
U3NT & 50 & 50 & -3 & Plateau-like Taiwan & On & North \\
U1.5N & 50 & 50 & -1.5 & Taiwan & &
\end{tabular}

typhoon was already deviating from its oceanic control during the period between -11 and $-8 \mathrm{~h}$. Its HA vector rotated $17^{\circ}$ clockwise relative to OC's, and its magnitude was somewhat larger (about $3.6 \mathrm{~m} \mathrm{~s}^{-1}$ ). The other terms were insignificant during this period.

The VA and DH terms, however, became more important as the U3N typhoon approached the coast. While the HA contribution remained directed northwestward, both DH and VA developed southwestward components (of 3 to $2.5 \mathrm{~m} \mathrm{~s}^{-1}$, respectively) in the 3 -h period prior to

(a)

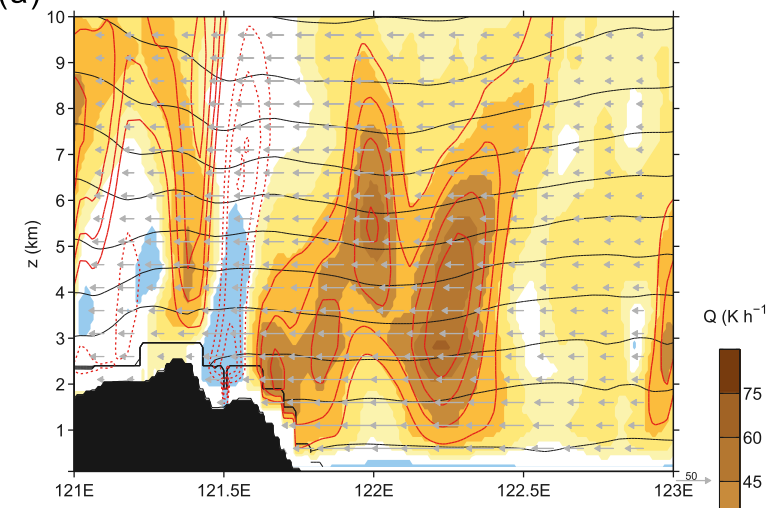

(b)

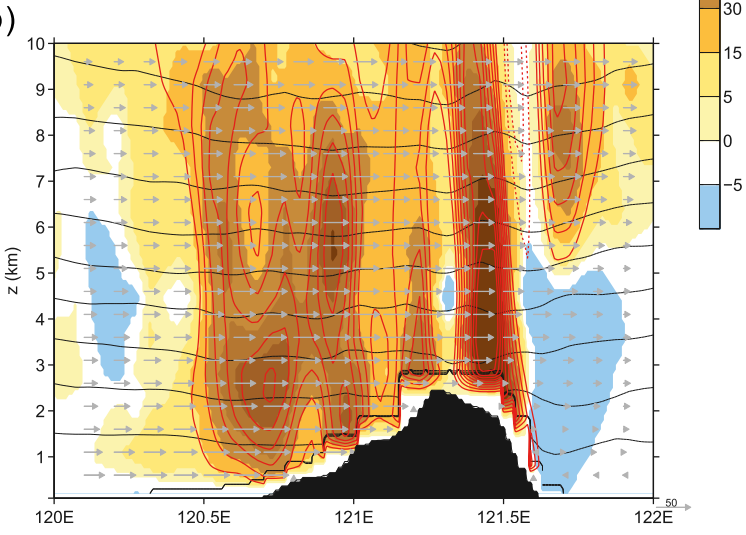

FIG. 5. Temporal mean cross sections of diabatic heating $Q$ (shaded), potential temperature (black contours), vertical velocities (red contours), and horizontal winds (vectors) over (a) $35-38 \mathrm{~h}$ for cross section a and (b) 51-54 h for cross section b, as indicated in Fig. 4. landfall, consistent with the typhoon's cyclonic (leftward) deviation and approximately $25-\mathrm{km}$ storm center displacement during this time. In the case of U3S, the DH and VA contributions were qualitatively similar but quantitatively smaller, in agreement with that typhoon's much more modest direction and speed change. This PV

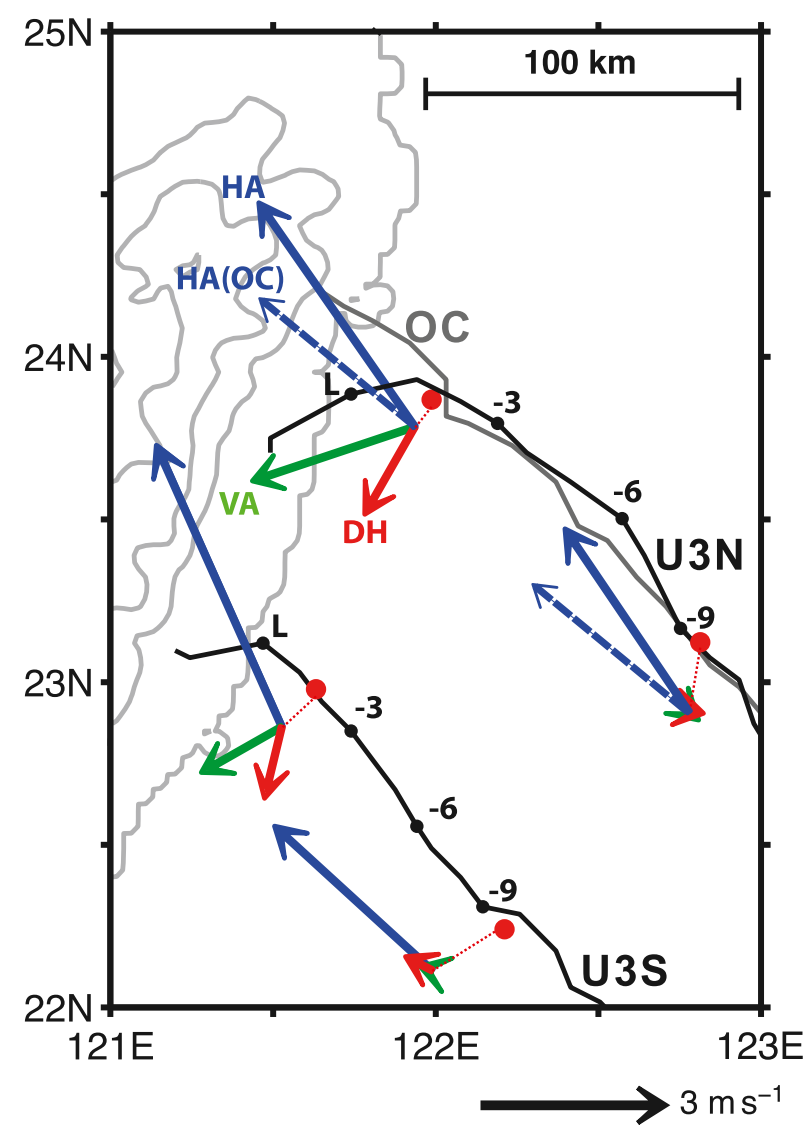

FIG. 6. The hourly tracks of the U3N and U3S experiments (black) and OC experiment (gray), annotated with hours prior to landfall $(L)$ expressed as negative numbers. The PV tendency contributions of HA, VA, and DH are plotted as vectors in blue, green, and red, respectively. The track of U3S's OC counterpart parallels that of the $\mathrm{U} 3 \mathrm{~N}$ case and thus is omitted for clarity. The DH and VA vectors of the OC case are not shown, as the magnitudes are too small. 
(a)

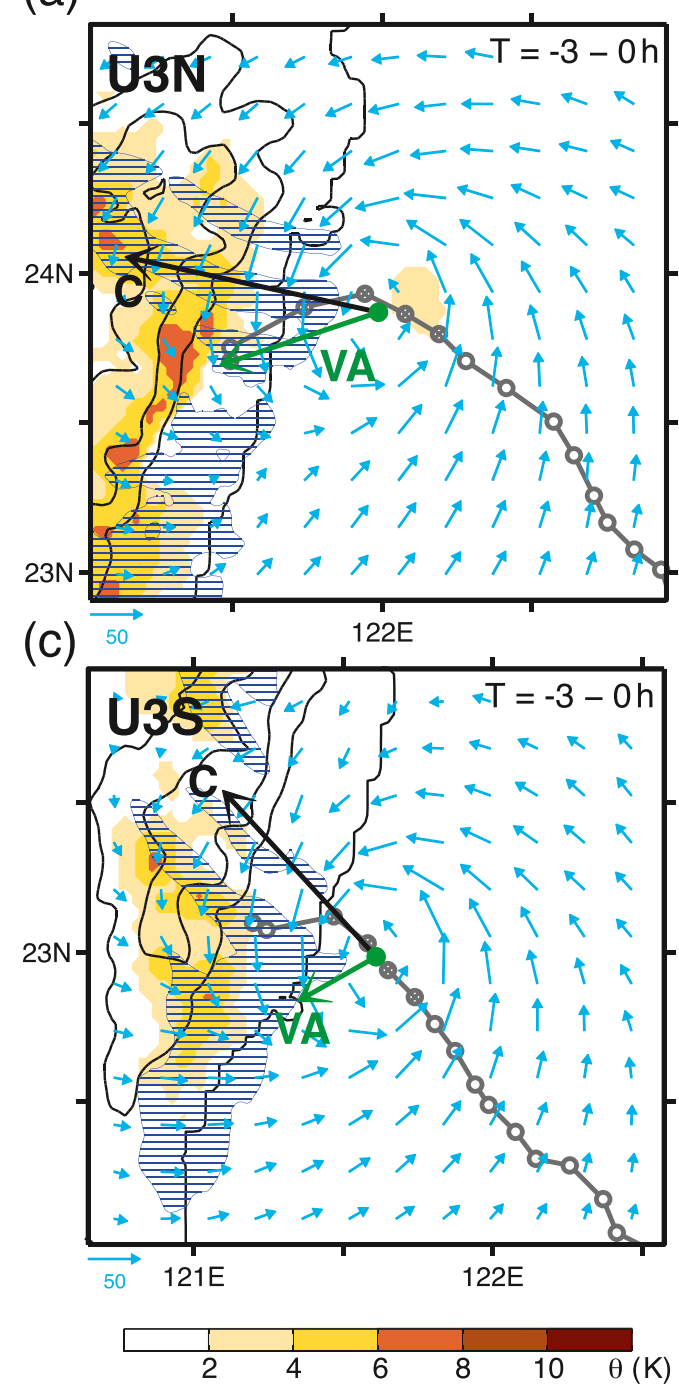

(b)

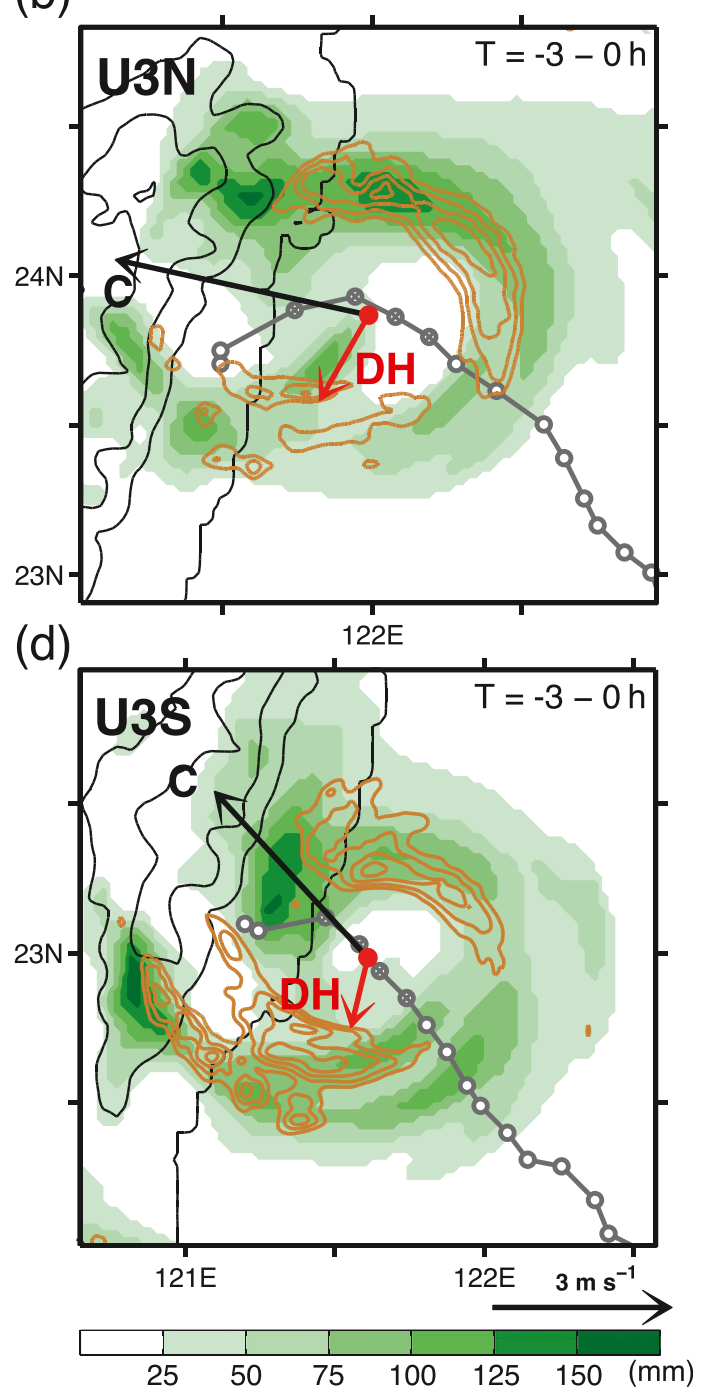

FIG. 7. Vortex-following composite fields from $-3 \mathrm{~h}$ to landfall for experiments U3N and U3S, with typhoon motion vector C (black), and VA (green) and DH (red) contributions to storm motion shown. (a),(c) Potential temperature anomalies (with respect to the initial time; shaded), downward motions (less than $-0.2 \mathrm{~m} \mathrm{~s}^{-1}$; hatched), and wind vectors at the lowest model level for U3N and U3S, respectively. (b),(d) Accumulated rainfall (shaded) and vertically averaged diabatic heating $Q$ (orange, contoured at $12,15,18$, and $21 \mathrm{~K} \mathrm{~h}^{-1}$ ) for $\mathrm{U} 3 \mathrm{~N}$ and $\mathrm{U} 3 \mathrm{~S}$.

tendency diagnosis result is also qualitatively consistent with the previous study of Tang and Chan (2014).

Figure 7 presents selected vortex-following composite fields from $t=-3 \mathrm{~h}$ to landfall for experiments U3N and U3S. Shown are the storm motion vector (labeled C) and potential temperature anomaly (relative to the initial state) and downward motion (left column) and total period rainfall and vertically averaged $Q$ (right column). The vector of total PV tendency (summations of HA, $\mathrm{VA}$, and $\mathrm{DH}$ ) is close to the storm motion vector in our analysis. Rather than being blocked, the circulation of U3N forced downward motion on the east-facing slope of the CMR (Fig. 7a), increasing the surface potential temperature by as much as $7.3 \mathrm{~K}$ southwest of the typhoon center, in agreement with Chen et al. (2010). Note that there was diminished rainfall and latent heating (Fig. 7b) where the descent was largest, as the subsiding motion logically suppressed convective activity, and the asymmetric distribution pattern of convection resembled the cases presented in Fig. 2.

Owing to the subsidence, the U3N case's $Q$ field was also quite asymmetric, which permitted the $\mathrm{DH}$ term to modulate this TC's motion. Note that the $\mathrm{DH}$ vector pointed toward a local minimum of convective heating and rainfall. 
H13 explained how this occurs (see p. 1015): in the northeast quadrant, the heating maximum was in the lower troposphere, causing its vertical gradient (the most important component of $\mathrm{DH}$ ) to be negative, while in the southwest quadrant, the heating was much smaller. As a consequence, the WN1 component of DH pointed southwest, away from its relative minimum and toward the relative maximum.

The topography also more directly contributes to U3N's asymmetry through the subsidence-caused vertical stretching that appears in the VA term $[-w(\partial P / \partial z)]$ of (3). The negative vertical velocity and positive PV vertical gradient in the lower levels created the positive VA contribution of PV tendency. In combination, the DH and VA contributions explain not only the U3N storm's DT (the turning to the left as the storm makes landfall), but also the absence of such deflection in the southerly landfall case. In case U3S, the subsidence (as manifested through the warming) was smaller (Fig. 7c), and the convective activity was less asymmetric (Fig. 7d), leading to smaller influences from the VA and DH terms. Note that because the HA vector also shifted during this period (Fig. 6), the combined effect of advection and diabatic forcing was to keep this storm moving in a nearly straight course as it approached the Taiwan coast.

As noted in our previous work (H13; Cao et al. 2011), the terms in this PV tendency decomposition are not independent. A change in diabatic heating, for example, directly and indirectly causes PV advection to respond, which could cause further adjustments in DH. One way of isolating the influence of $\mathrm{DH}$, however, might be to deactivate diabatic forcing from microphysics prior to the development of significant track deflection. Thus, we create experiments NDH and OCNDH (Fig. 8), which are versions of U3N and OC in which microphysics is switched off $4 \mathrm{~h}$ prior to landfall, before significant asymmetries appeared in the $Q$ field, and integrated a further $6 \mathrm{~h}$.

Without microphysics forcing, TCs will eventually spin down, and in the oceanic control version, storm OCNDH did weaken relative to OC (not shown), but note that its motion was little affected (Fig. 8a). Similarly, case NDH also weakened relative to $\mathrm{U} 3 \mathrm{~N}$, and its central pressure rose from $965 \mathrm{hPa}$ at deactivation time to $981 \mathrm{hPa}$ at its eventual landfall (compared to $970 \mathrm{hPa}$ for U3N). Importantly, however, without asymmetric $\mathrm{DH}$, typhoon $\mathrm{NDH}$ failed to turn as case U3N did. Instead, its cyclonic turn was delayed until its essentially unmodified motion could carry it physically closer to the coastline.

At that point, the VA contribution finally emerged as significant in case NDH. This is illustrated in Fig. 8b, which presents PV tendency component vectors for $\mathrm{NDH}$ and $\mathrm{U} 3 \mathrm{~N}$, representing conditions just prior to the onset of the former case's leftward deflection. This figure demonstrates that vertical stretching induced by

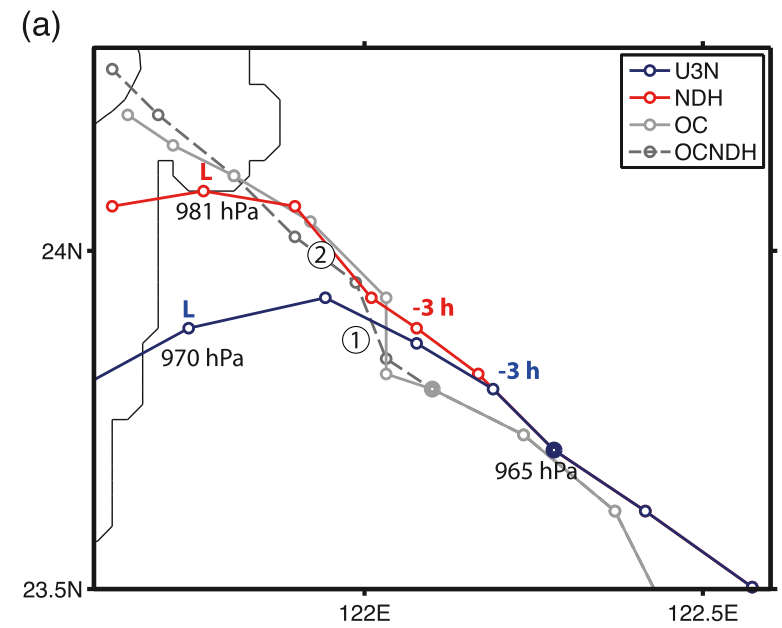

(b) -3 - $0 \mathrm{~h}$ PV tendency diagnosis
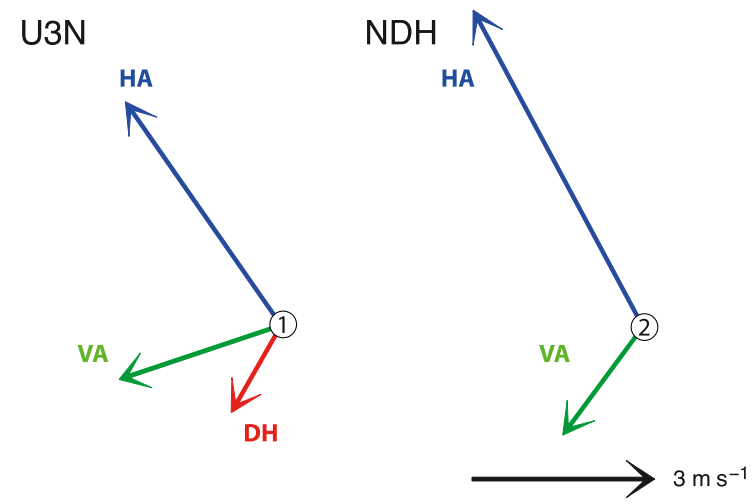

FIG. 8. (a) Hourly tracks from the sensitivity experiment with diabatic heating turned off (NDH; red) at the time denoted by the bold blue circle, with the control U3N storm track shown for reference. (b) The PV tendency diagnosis from $-3 \mathrm{~h}$ to landfall for the U3N 1 and NDH 2 experiments.

topography alone suffices to induce DTs. That said, this experiment also reveals that terrain-induced heating asymmetries are important and play a crucial role in storm U3N's deflection when and where it does occur.

\section{c. Sensitivity tests}

Figure 9 presents tracks from versions of the U3N experiment in which the initial vortex was either made weaker or larger in size (see Table 2). Starting with a weaker vortex (case U3NV15; brown) slowed the storm's development, permitting a larger westward drift that helped shift the landfall location southward relative to U3N. Similar to case U3S, the contributions of VA and DH to U3NV15's motion were small, consistent with the absence of deflection as the TC arrived at the coastline. Convective activity as revealed via precipitation and $Q$ was not very asymmetric (Fig. 10b), and the weak storm circulation was blocked by the CMR, leading to little in the way of downslope warming (Fig. 10a). 


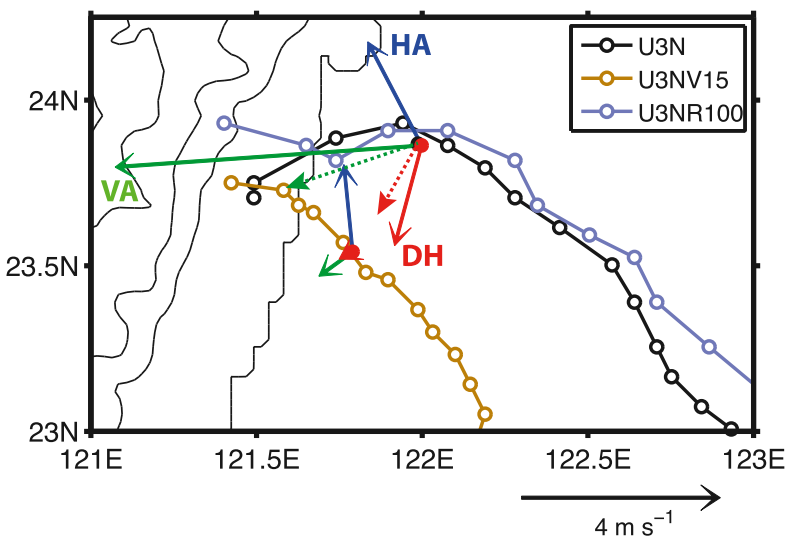

FIG. 9. Hourly tracks of weaker initial storm $\left(V_{m}=15 \mathrm{~m} \mathrm{~s}^{-1}\right.$, experiment U3NV15, orange) and larger initial storm $\left(R_{m}=100 \mathrm{~km}\right.$, experiment U3NR100, light blue), with HA (blue arrow), VA (green arrow), and DH (red arrow) contributions to storm motion shown, along with those from U3N experiment (dashed arrows) for comparison. The HA vector of $\mathrm{U} 3 \mathrm{~N}$ is not shown for clarity.

In contrast, expanding the initial vortex (case U3NR100; light blue) was found to increase the storm speed, albeit with little alteration in track. Relative to U3N, the broader circulation of U3NR100 resulted in even more vigorous (and more asymmetric) convection, as well as more significant downslope warming (Figs. 10c,d) as the storm approached the CMR. As a consequence, both DH and VA contributions to the PV tendency were larger, especially the latter, which exceeded U3N's by $0.7 \mathrm{~m} \mathrm{~s}^{-1}$. For convenience, U3N's VA and DH vectors are shown with dashed arrows; the HA vectors point northwestward in all cases (Figs. 6, 9).

To further examine the effect of topographic blocking on cyclonic track deflection, experiment U3NT (see Table 2) utilizes a terrain that is modified by this empirically selected function:

$$
h_{\text {new }}=h_{\text {taiwan }}\left(4-0.0008 h_{\text {taiwan }}\right) \text {, }
$$

where $h_{\text {taiwan }}$ is the original Taiwan terrain height in $\mathrm{km}$. This transformation makes the model terrain higher and sharper with an extensive plateau but otherwise retains the general features of the original Taiwan topography (Fig. 11). In contrast to case U3N, note that the modified terrain storm U3NT executed a northward (rightward) deflection upon approaching the coastline (Fig. 11a). This appears to be a consequence of both reduced $\mathrm{DH}$ (Fig. 11a) and substantially altered VA (Fig. 11b) contributions to the PV tendencies that occur as storm U3NT approached the coastline.

First, we note that on cyclone U3NT's north flank, the vertical motion and diabatic heating fields (Fig. 11b) were more vigorous compared to case $\mathrm{U} 3 \mathrm{~N}$, with maxima residing at higher altitudes (cf. Figs. 11c and 5a). As a result, the vertical gradient of $Q$ averaged through the lower troposphere was less negative in this quadrant of this TC, which actually acts to reduce the asymmetry of the DH term. Therefore, one contributing factor to case U3N's DT has been substantially decreased in magnitude. At the same time, note the VA vector for this case was directed northwestward and, thus, augmented rather than competed with HA. This appears to occur in this situation owing to the greater blocking of the TC circulation by the higher and steeper terrain. Note that the subsidence appeared on the northwest side of the storm, which helped alter the VA direction, as illustrated in Fig. 11b.

Figure 12 presents moist Froude numbers (Fr; contours) as well as vertically averaged horizontal winds (vectors) and wind speeds (shadings) for the U3N, U3NV15, and U3NT experiments. Since we focus on the flow in the lower levels that may be altered by the model terrain, the horizontal wind speeds have been vertically averaged over the lowest 12 model levels (to about $3.5 \mathrm{~km}$ above the surface, which corresponds to the tallest peaks.). Following Chen and Lin (2005) and Chen et al. (2008), the moist Froude number is defined as $\mathrm{Fr}=U /\left(N_{w} h\right)$, where $U$ is horizontal wind speed, $h$ is the terrain height, and the unsaturated moist BruntVäisälä frequency is $N_{w}^{2}=\left(g / \theta_{v}\right)\left(\partial \theta_{v} / \partial z\right)$, with $\theta_{v}$ being the virtual potential temperature. The Froude number concept suggests that the flow can pass over the mountain barrier when and where $\mathrm{Fr}$ is greater than one, which is favored by higher wind speeds and/or lower vertical stabilities. In Fig. 12, Fr $=1$ contours corresponding to presumed $U$ values of 15 (solid), 30 (dashed), and $45 \mathrm{~m} \mathrm{~s}^{-1}$ (dotted), respectively, are provided for reference, and vectors are only shown where wind speeds exceed these values.

In the U3N experiment (Fig. 12a), the low-level wind speeds generally exceeded $30 \mathrm{~m} \mathrm{~s}^{-1}$ in the area where $\mathrm{Fr}$ was greater than 1 when presumed speeds $\geq 15 \mathrm{~m} \mathrm{~s}^{-1}$ (i.e., within the area enclosed by the solid black contour). Thus, the low-level flow was generally strong enough relative to the vertical stability to pass over the mountain range, and the stretching effect became significant. However, when the circulation was weak, as in the U3NV15 case (Fig. 12b), the low-level flow was largely blocked. There was corresponding less vertical stretching, leading to the reduction in the VA contribution to storm motion seen previously (Fig. 10a).

A similar situation occurs in the U3NT case (Fig. 12c), in which larger wind speeds were needed to surmount this case's modified topography, and this occurred only on the island's northern and southern tips. Thus, the stretching effect was concentrated on the storm's northwest flank, which caused the VA contribution to shift in direction. This, combined with 
(a)
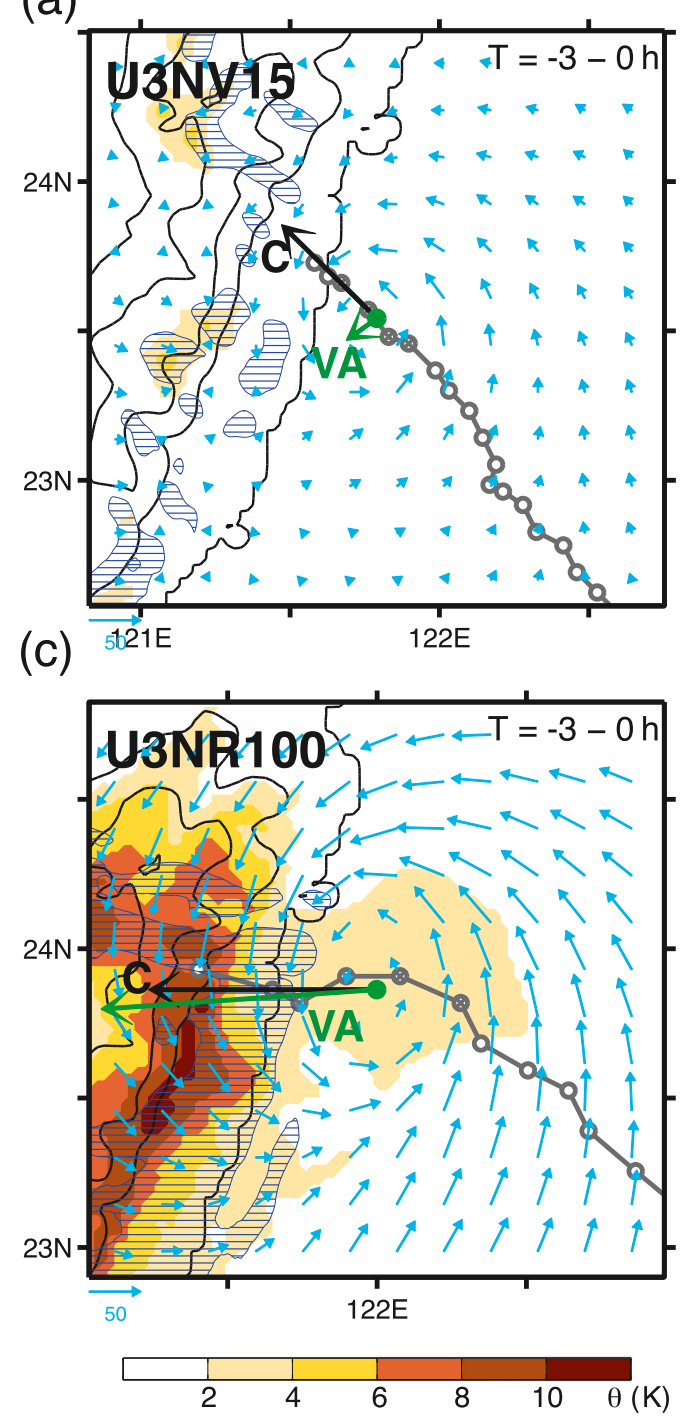

(b)
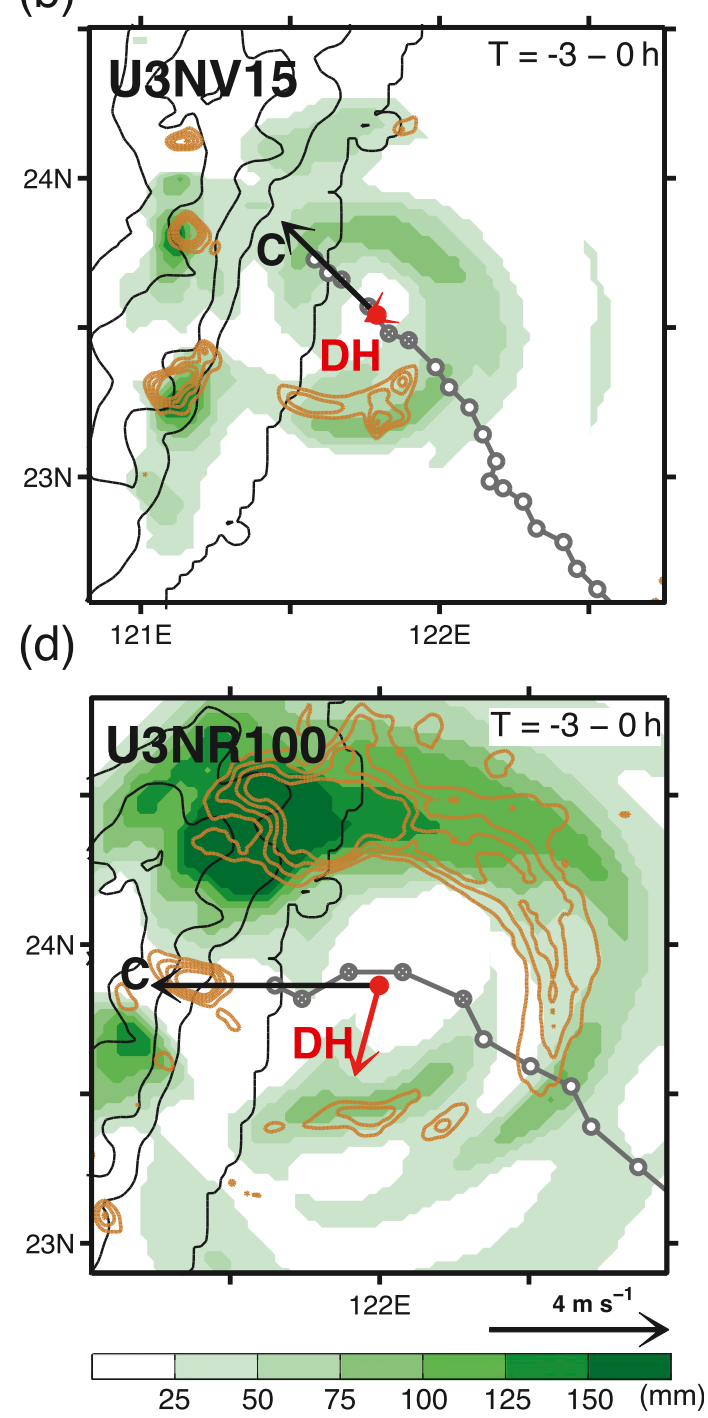

FIG. 10. As in Fig. 7, but for the (a),(b) U3NV15 and (c),(d) U3NR100 experiments.

the reduced convective asymmetry that drives the DH term, resulted in a rightward-turning DT upon approaching land.

Finally, we consider experiment U1.5N (Table 2, Fig. 13), which imposed weaker $\left(-1.5 \mathrm{~m} \mathrm{~s}^{-1}\right)$ initial easterly winds. It is immediately apparent that the track deflection became even more significant with the slower motion, which gave the typhoon more time to be influenced by the vertical stretching and convective diabatic heating effects, owing to its winds encountering the topography. As a consequence, the track deflection commenced even farther from the coastline, prolonging the period prior to landfall even more. Previous studies have shown that DTs can extend typhoon duration time in the prelandfall phase and thus may induce more extreme precipitation before the TC makes landfall (Su et al. 2012).

\section{Conclusions}

We analyzed 84 typhoons that reached the mountainous island of Taiwan's east coast in the past 51 years from 1960 to 2010. Typhoons with "deflection tracks" (DTs), or significant motion deviations within a 200-km distance from the east coast, were examined. There were $49 \mathrm{DT}$ cases that turned to the left-hand side (toward the southwest) prior to landfall, including 18 with very large deflection angles (DA $>20^{\circ}$ ), and another seven had looped tracks (LTs). These deflections postponed the typhoons' landfalls and prolonged the duration of rainfall in Taiwan. Most of the 
(a)

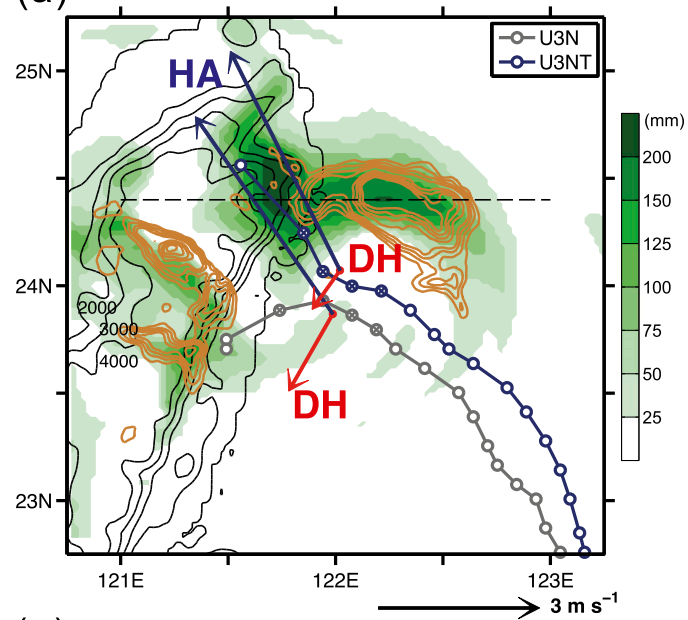

(b)

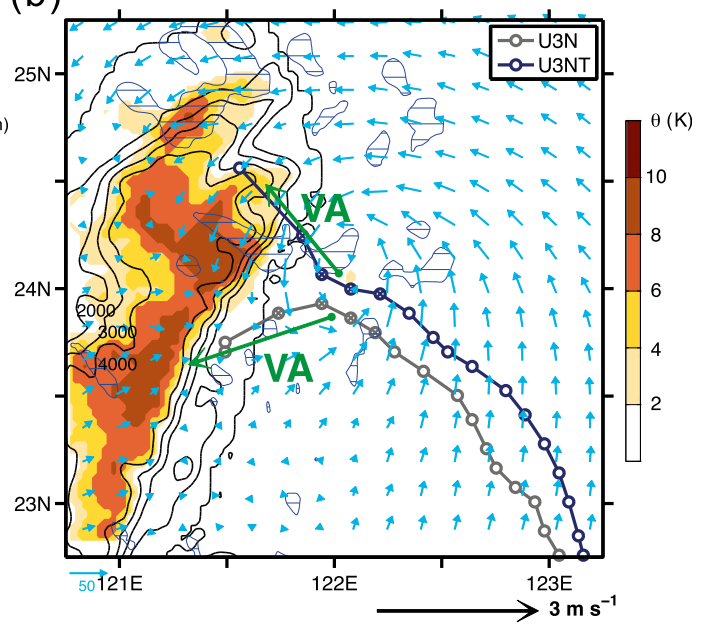

(c)

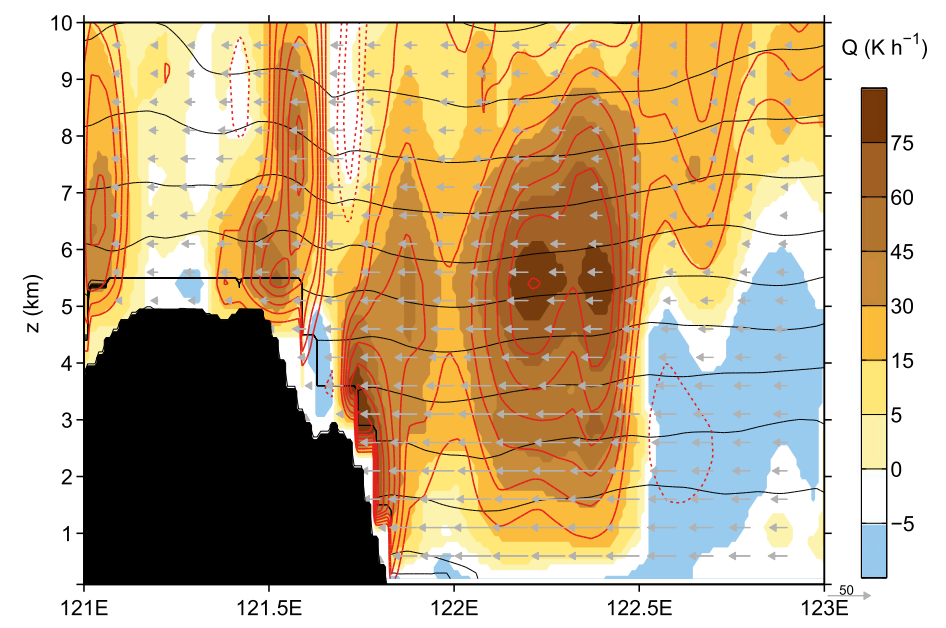

FIG. 11. Results from terrain sensitivity experiment U3NT, which shares model settings with U3N but with a modified, plateau-type Taiwan topography (see text). (a) Rainfall (shaded) and vertically averaged diabatic heating $Q$ similar to Fig. 7b, but superposed with U3NT tracks (blue) and HA (blue vector) and DH (red vector) contributions for simulations U3NT and U3N. The vertically averaged diabatic heating $Q$ (orange) is contoured at $12,15,18,21,27,30,33$, and $36 \mathrm{~K} \mathrm{~h}^{-1}$. (b) Potential temperature anomalies for U3NT, as in Fig. 7a. (c) As in Fig. 5, but from $-3 \mathrm{~h}$ to landfall for U3NT. The cross-sectional location is indicated by the dashed line in (a).

large DA and LT cases reached Taiwan's east coast poleward of $24^{\circ} \mathrm{N}$, provoked localized subsidence warming associated with downslope flow on their southwestern sides, and exhibited southward track displacements of 11-26 km. The relative rarity of DTs among storms making landfall south of $24^{\circ} \mathrm{N}$ suggested to us that mechanisms other than terrain blocking and the channeling effect may be needed to explain the DT phenomenon.

Thus, we designed and performed idealized WRF simulations of DT cases, along with potential vorticity (PV) tendency diagnoses of storm motion. The simulated typhoons encountered a Taiwan-like obstacle composed of water. The track deflections were interpreted via the contributions of the horizontal and vertical advection
(HA and VA) and diabatic heating (DH) PV tendencies. Our control experiment (U3N) exhibited a leftwardturning DT at $3 \mathrm{~h}$ prior to making "landfall" on the east coast, but the HA term did not directly contribute to this southwestward track deflection. Instead, it was the VA and DH contributions that provided the roughly $25-\mathrm{km}$ displacement, the magnitude of which is in general agreement with those observed for large DA cases.

Except for the very weak storm (U3NV15) and very tall mountain (U3NT) experiments, the local Froude (Fr) numbers exceeded 1, and thus, the flow could cross over the mountain to produce an asymmetric local PV source in the VA term. In the Fr $>1$ situation, the flow to the south of a storm could cross the steep terrain and 
(a) U3N

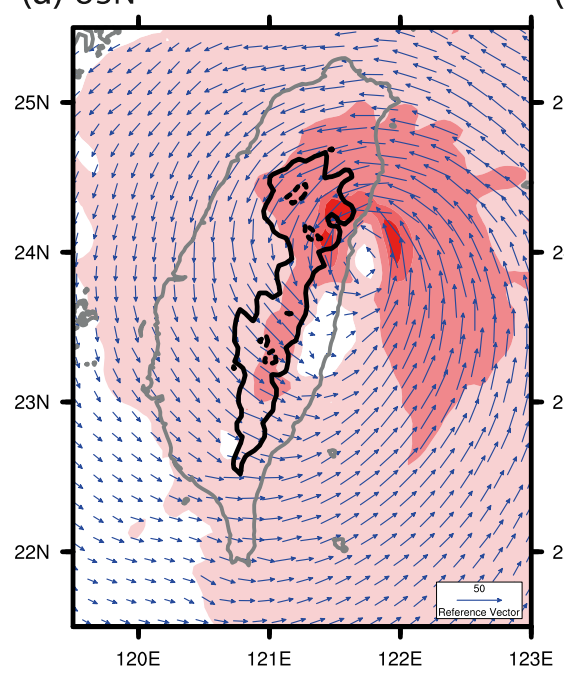

(b) U3NV15

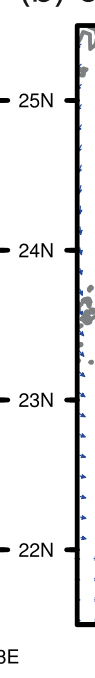

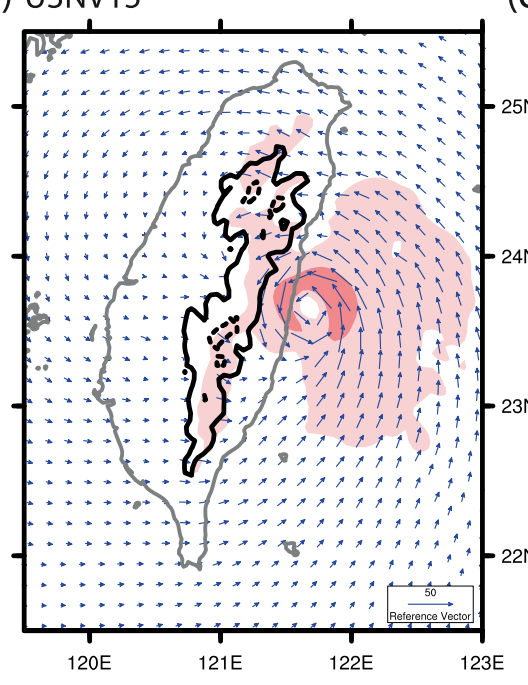

(c) U3NT

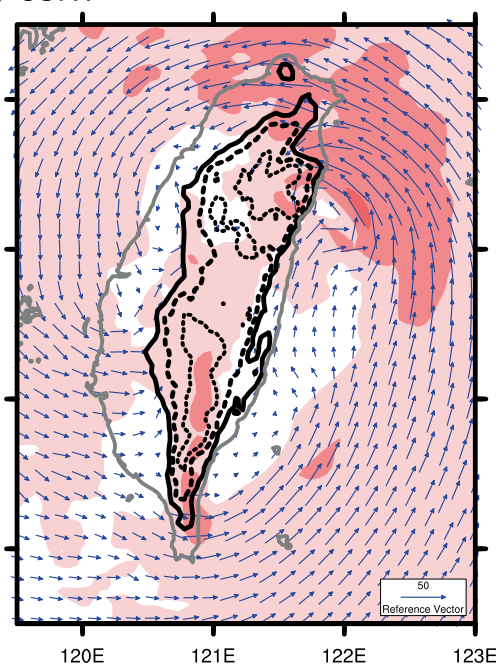

15 30
Wind speed $\left(\mathrm{m} \mathrm{s}^{-1}\right)$

FIG. 12. Vertically averaged horizontal winds (vectors), wind speed (shaded), and moist Froude numbers (contours) over model levels 1-12 (up to about 3.5-km height) at landfall time for the (a) U3N, (b) U3NV15, and (c) U3NT experiments. The moist Froude number is given by $\mathrm{Fr}=U /\left(N_{w} h\right)$ (see text), and $\mathrm{Fr}=1$ is plotted presuming $U$ equals to 15 (solid), 30 (dashed), and $45 \mathrm{~m} \mathrm{~s}^{-1}$ (dotted), respectively. For clarity, the wind vectors are only shown where Fr suggests the wind speed can pass over the terrain.

cause significant vortex stretching, which appears in the PV tendency's VA term, helping to shift the storm southward from its original northwestward heading. This leads to the large DA typhoons. The vortex stretching was most significant for TCs approaching the northern part of the island, in agreement with the observation that most large DA cases have occurred on the northern part of Taiwan's east coast.

The cross-mountain subsidence also suppresses convection on the southwestern side of the storms as they approach the Taiwan east coast, establishing asymmetry in the diabatic forcing within the typhoons

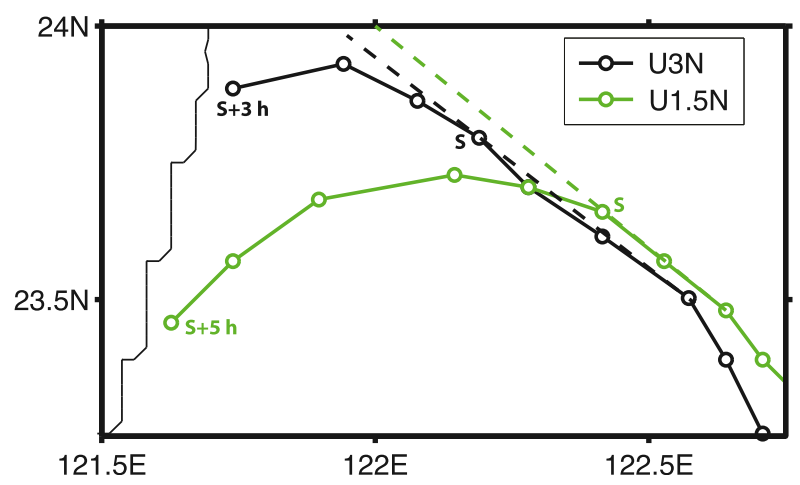

FIG. 13. Hourly positions for the slow-moving TC experiment U1.5N (green) as it was approaching Taiwan, for comparison with control run U3N. The points marked $\mathrm{S}$ are the TC starting defection points. Note that $\mathrm{U} 1.5 \mathrm{~N}$ has more time to deflect southward than U3N. that directly affects storm motion through the PV equation's DH term. For TCs approaching the northern part of the island, this asymmetry contributed to the storms' southward deflections prior to landfall, in a manner similar to that examined in H13. For storms approaching southern Taiwan, however, the vortex stretching was not nearly as significant, and convective activity remained more symmetric, and thus, much less deflection resulted. Our results highlight the importance of cross-mountain, flow-induced vortex stretching and asymmetric diabatic heating to typhoon deflection tracks.

Acknowledgments. This research was supported by the Ministry of Science and Technology, Taiwan, under Grants MOST 106-2111-M-492-003-, MOST 105-2111M-492-001-MY2, MOST 106-2111-M-034-005-, MOST 105-2111-M-034-003-, MOST 106-2119-M-002-016-, MOST 104-2111-M-002-002-MY3-, and the U.S. Naval Research Laboratory under Grant N62909-15-1-2008. We also thank the Central Weather Bureau typhoon database (http://rdc28.cwb.gov.tw/) for providing the typhoon data.

\section{REFERENCES}

Bender, M. A., R. E. Tuleya, and Y. Kurihara, 1987: A numerical study of the effect of island terrain on tropical cyclones. Mon. Wea. Rev., 115, 130-155, https://doi.org/10.1175/1520-0493 (1987)115<0130:ANSOTE >2.0.CO;2. 
Brand, S., and J. Blelloch, 1974: Changes in the characteristics of typhoons crossing the island of Taiwan. Mon. Wea. Rev., 102, 708-713, https://doi.org/10.1175/1520-0493(1974)102<0708: CITCOT $>2.0 . \mathrm{CO} ; 2$.

Cao, Y., R. G. Fovell, and K. L. Corbosiero, 2011: Tropical cyclone track and structure sensitivity to initialization in idealized simulations: A preliminary study. Terr. Atmos. Oceanic Sci., 22, 559-578, https://doi.org/10.3319/TAO.2011.05.12.01(TM).

Carnevale, G. F., G. K. Vallis, R. Purini, and M. Briscolini, 1988 Propagation of barotropic modons over topography. Geophys. Astrophys. Fluid Dyn., 41, 45-101, https://doi.org/10.1080/ 03091928808208831.

_ , R. C. Kloosterziel, and G. J. F. van Heijst, 1991: Propagation of barotropic vortices over topography in a rotating tank. J. Fluid Mech., 233, 119-139, https://doi.org/10.1017/ S0022112091000411.

Chan, J. C. L., F. M. F. Ko, and Y. M. Lei, 2002: Relationship between potential vorticity tendency and tropical cyclone motion. J. Atmos. Sci., 59, 1317-1336, https://doi.org/10.1175/ 1520-0469(2002)059<1317:RBPVTA $>2.0 . C O ; 2$.

Chang, C.-P., Y.-T. Yang, and H.-C. Kuo, 2013: Large increasing trend of tropical cyclone rainfall in Taiwan and the roles of terrain. J. Climate, 26, 4138-4147, https://doi.org/10.1175/ JCLI-D-12-00463.1.

Chang, S. W.-J., 1982: The orographic effects induced by an island mountain range on propagating tropical cyclones. Mon. Wea. Rev., 110, 1255-1270, https://doi.org/10.1175/ 1520-0493(1982)110<1255:TOEIBA $>2.0$. CO;2.

Chen, S.-H., and Y.-L. Lin, 2005: Effects of moist Froude number and CAPE on a conditionally unstable flow over a mesoscale mountain ridge. J. Atmos. Sci., 62, 331-350, https://doi.org/ 10.1175/JAS-3380.1.

- - — - and Z. Zhao, 2008: Effects of unsaturated moist Froude number and orographic aspect ratio on a conditionally unstable flow over a mesoscale mountain. J. Meteor. Soc. Japan, 86, 353-367, https://doi.org/10.2151/jmsj.86.353.

Chen, T.-C., S.-Y. Wang, M.-C. Yen, A. J. Clark, and J.-D. Tsay, 2010: Sudden surface warming-drying events caused by typhoon passages across Taiwan. J. Appl. Meteor. Climatol., 49, 234-252, https://doi.org/10.1175/2009JAMC2070.1.

Chiou, T.-K., C.-R. Chen, P.-L. Chang, and P.-F. Lin, 2006: Quantitative precipitation estimation using multiple sensors (in Chinese). 2006 Conf. on Weather Analysis and Forecasting, Tao-Yuan, Taiwan, Central Weather Bureau, 5-28.

Dudhia, J., 1989: Numerical study of convection observed during the Winter Monsoon Experiment using a mesoscale twodimensional model. J. Atmos. Sci., 46, 3077-3107, https://doi.org/ 10.1175/1520-0469(1989)046<3077:NSOCOD>2.0.CO;2.

Fovell, R. G., and H. Su, 2007: Impact of cloud microphysics on hurricane track forecasts. Geophys. Res. Lett., 34, L24810, https://doi.org/10.1029/2007GL031723.

_, K. L. Corbosiero, and H. C. Kuo, 2009: Cloud microphysics impact on hurricane track as revealed in idealized experiments. J. Atmos. Sci., 66, 1764-1778, https://doi.org/10.1175/ 2008JAS2874.1.

$\longrightarrow,-$ A. Seifert, and K.-N. Liou, 2010: Impact of cloudradiative processes on hurricane track. Geophys. Res. Lett., 37, L07808, https://doi.org/10.1029/2010GL042691.

—_, Y. P. Bu, K. L. Corbosiero, W.-W. Tung, Y. Cao, H.-C. Kuo, L.-H. Hsu, and H. Su, 2016: Influence of cloud microphysics and radiation on tropical cyclone structure and motion. Multiscale Convection-Coupled Systems in the Tropics: A Tribute to Dr. Michio Yanai, Meteor. Monogr.,
No. 56, Amer. Meteor. Soc., 11.1-11.27, https://doi.org/ 10.1175/AMSMONOGRAPHS-D-15-0006.1.

Hong, S.-Y., 2010: A new stable boundary-layer mixing scheme and its impact on the simulated East Asian summer monsoon. Quart. J. Roy. Meteor. Soc., 136, 1481-1496, https://doi.org/ 10.1002/qj.665.

Hsu, L.-H., H.-C. Kuo, and R. G. Fovell, 2013: On the geographic asymmetry of typhoon translation speed across the mountainous island of Taiwan. J. Atmos. Sci., 70, 1006-1022, https:// doi.org/10.1175/JAS-D-12-0173.1.

Huang, C.-Y., and Y.-L. Lin, 2008: The influence of mesoscale mountains on vortex tracks: Shallow-water modeling study. Meteor. Atmos. Phys., 101, 1-20, https://doi.org/10.1007/ s00703-007-0284-1.

Huang, Y.-H., C.-C. Wu, and Y. Wang, 2011: The influence of island topography on typhoon track deflection. Mon. Wea. Rev., 139, 1708-1727, https://doi.org/10.1175/2011MWR3560.1.

Jian, G.-J., and C.-C. Wu, 2008: A numerical study of the track deflection of Supertyphoon Haitang (2005) prior to its landfall in Taiwan. Mon. Wea. Rev., 136, 598-615, https://doi.org/ 10.1175/2007MWR2134.1.

Jordan, C. L., 1958: Mean soundings for the West Indies area. $J$. Meteor., 15, 91-97, https://doi.org/10.1175/1520-0469(1958)015<0091: MSFTWI $>2.0 . \mathrm{CO} ; 2$

Kuo, H.-C., R. T. Williams, J.-H. Chen, and Y.-L. Chen, 2001: Topographic effects on barotropic vortex motion: No mean flow. J. Atmos. Sci., 58, 1310-1327, https://doi.org/10.1175/ 1520-0469(2001)058<1310:TEOBVM >2.0.CO;2.

Lin, Y.-L., and L. C. Savage III, 2011: Effects of landfall location and the approach angle of a cyclone vortex encountering a mesoscale mountain range. J. Atmos. Sci., 68, 2095-2106, https://doi.org/10.1175/2011JAS3720.1.

, R. D. Farley, and H. D. Orville, 1983: Bulk parameterization of the snow field in a cloud model. J. Climate Appl. Meteor., 22, 1065-1092, https://doi.org/10.1175/1520-0450 (1983) $022<1065$ :BPOTSF $>2.0 . \mathrm{CO} ; 2$.

—, J. Han, D. W. Hamilton, and C.-Y. Huang, 1999: Orographic influence on a drifting cyclone. J. Atmos. Sci., 56, 534-562, https://doi.org/10.1175/1520-0469(1999)056<0534: OIOADC $>2.0 . \mathrm{CO} ; 2$.

— S.-Y. Chen, C. M. Hill, and C.-Y. Huang, 2005: Control parameters for the influence of a mesoscale mountain range on cyclone track continuity and deflection. J. Atmos. Sci., 62, 1849-1866, https://doi.org/10.1175/JAS3439.1

, S.-H. Chen, and L. Liu, 2016: Orographic influence on basic flow and cyclone circulation and their impacts on track deflection of an idealized tropical cyclone. J. Atmos. Sci., 73, 3951-3974, https://doi.org/10.1175/JAS-D-15-0252.1.

Liu, L., Y.-L. Lin, and S.-H. Chen, 2016: Effects of landfall location and approach angle of an idealized tropical cyclone over a long mountain range. Front. Earth Sci., 4, 14, https://doi.org/ 10.3389/feart.2016.00014.

Mlawer, E. J., S. J. Taubman, P. D. Brown, M. J. Iacono, and S. A. Clough, 1997: Radiative transfer for inhomogeneous atmospheres: RRTM, a validated correlated-k model for the longwave. J. Geophys. Res., 102, 16663-16 682, https://doi.org/ 10.1029/97JD00237.

Shieh, S.-L., S.-T. Wang, M.-D. Cheng, and T.-C. Yeh, 1998: Tropical cyclone tracks over Taiwan and its vicinity for the one hundred years 1897 to 1996 (in Chinese). Central Weather Bureau Research Rep. CWB86-1M-01, 497 pp.

Su, S.-H., H.-C. Kuo, L.-H. Hsu, and Y.-T. Yang, 2012: Temporal and spatial characteristics of typhoon extreme rainfall in 
Taiwan. J. Meteor. Soc. Japan, 90, 721-736, https://doi.org/ 10.2151/jmsj.2012-510.

Tang, C. K., and J. C. L. Chan, 2014: Idealized simulations of the effect of Taiwan and Philippines topographies on tropical cyclone tracks. Quart. J. Roy. Meteor. Soc., 140, 1578-1589, https://doi.org/10.1002/qj.2240.

_, and - 2015: Idealized simulations of the effect of local and remote topographies on tropical cyclone tracks. Quart. J. Roy. Meteor. Soc., 141, 2045-2056, https://doi.org/10.1002/qj.2498.

_ and —, 2016: Idealized simulations of the effect of Taiwan topography on the tracks of tropical cyclones with different steering flow strengths. Quart. J. Roy. Meteor. Soc., 142, 32113221, https://doi.org/10.1002/qj.2902.

Wang, C.-C., H.-C. Kuo, Y.-H. Chen, H.-L. Huang, C.-H. Chung, and K. Tsuboki, 2012: Effects of asymmetric latent heating on typhoon movement crossing Taiwan: The case of Morakot (2009) with extreme rainfall. J. Atmos. Sci., 69, 3172-3196, https://doi.org/10.1175/JAS-D-11-0346.1.

, Y.-H. Chen, H.-C. Kuo, and S.-Y. Huang, 2013: Sensitivity of typhoon track to asymmetric latent heating/rainfall induced by Taiwan topography: A numerical study of Typhoon Fanapi (2010). J. Geophys. Res. Atmos., 118, 3292-3308, https://doi.org/ 10.1002 /jgrd.50351.
Wang, S.-T., 1980: Prediction of the behavior and intensity of typhoons in Taiwan and its vicinity (in Chinese). Chinese National Science Council Research Rep. 108, 100 pp.

Wu, C.-C., T.-H. Li, and Y.-H. Huang, 2015: Influence of mesoscale topography on tropical cyclone tracks: Further examination of the channeling effect. J. Atmos. Sci., 72, 3032-3050, https://doi.org/ 10.1175/JAS-D-14-0168.1.

Wu, L., and B. Wang, 2000: A potential vorticity tendency diagnostic approach for tropical cyclone motion. Mon. Wea. Rev., 128, 1899-1911, https://doi.org/10.1175/1520-0493(2000)128<1899: APVTDA $>2.0 . \mathrm{CO} ; 2$.

$\longrightarrow$, and — 2001: Effects of convective heating on movement and vertical coupling of tropical cyclones: A numerical study. J. Atmos. Sci., 58, 3639-3649, https://doi.org/10.1175/ 1520-0469(2001)058<3639:EOCHOM > 2.0.CO;2.

Yeh, T.-C., and R. L. Elsberry, 1993: Interaction of typhoons with the Taiwan orography. Part I: Upstream track deflections. Mon. Wea. Rev., 121, 3193-3212, https://doi.org/10.1175/ 1520-0493(1993)121<3193:IOTWTT>2.0.CO;2.

Zehnder, J. A., 1993: The influence of large-scale topography on barotropic vortex motion. J. Atmos. Sci., 50, 25192532, https://doi.org/10.1175/1520-0469(1993)050<2519: TIOLST $>2.0 . \mathrm{CO} ; 2$. 